\title{
Neutron star crust in Voigt approximation: general symmetry of the stress-strain tensor and an universal estimate for the effective shear modulus
}

\author{
Andrey I. Chugunov, ${ }^{1 \star}$ \\ ${ }^{1}$ Ioffe Institute, Politekhnicheskaya 26, 194021 St. Petersburg, Russia
}

Accepted XXX. Received YYY; in original form ZZZ

\begin{abstract}
I discuss elastic properties of neutron star crust in the framework of static Coulomb solid model when atomic nuclei are treated as non-vibrating point charges; electron screening is neglected. The results are also applicable for solidified white dwarf cores and other materials, which can be modeled as Coulomb solids (dusty plasma, trapped ions, etc.). I demonstrate that the Coulomb part of the stress-strain tensor has additional symmetry: contraction $B_{i j i l}=0$. It does not depend on the structure (crystalline or amorphous) and composition. I show as a result of this symmetry the effective (Voigt averaged) shear modulus of the polycrystalline or amorphous matter to be equal to $-2 / 15$ of the Coulomb (Madelung) energy density at undeformed state. This result is general and exact within the model applied. Since the linear mixing rule and the ion sphere model are used, I can suggest a simple universal estimate for the effective shear modulus: $\sum_{Z} 0.12 n_{Z} Z^{5 / 3} e^{2} / a_{\mathrm{e}}$. Here summation is taken over ion species, $n_{Z}$ is number density of ions with charge $Z e$. Finally $a_{\mathrm{e}}=\left(4 \pi n_{\mathrm{e}} / 3\right)^{-1 / 3}$ is electron sphere radius. Quasineutrality condition $n_{\mathrm{e}}=\sum_{Z} Z n_{Z}$ is assumed.
\end{abstract}

Key words: stars: neutron - white dwarfs - stars: oscillations

\section{INTRODUCTION}

A neutron star crust as well as a white dwarf core is made of fully ionized atomic nuclei (ions) and almost unpolarizable background of degenerate electrons (e.g., Haensel et al. 2006; Chamel \& Haensel 2008; Caplan \& Horowitz 2017; Rezzolla et al. 2018). At low enough temperature, which is typical for neutron stars and white dwarfs at their late evolutionary stages, ions solidify (e.g., Brush et al. 1966; Ogata et al. 1993; Jones \& Ceperley 1996; Potekhin \& Chabrier 2000; Caplan \& Horowitz 2017; Medin \& Cumming 2010; Caplan et al. 2018). If there is only one type of atomic nuclei at a given layer, they form a crystal with a body-centered cubic (bcc) lattice (Hamaguchi et al. 1997; Baiko 2002; Kozhberov 2018). However, in multicomponent models (e.g., Fantina et al. 2020; Carreau et al. 2020a,b) more complicated lattices are possible (e.g., Ogata et al. 1993; Chamel \& Fantina 2016; Kozhberov 2019). Solidification into amorphous structure is discussed elsewhere (e.g., Jones 1999; Sauls et al. 2020).

In solidified matter the shear stresses can be maintained by elasticity, which is important for physics of neutron stars (e.g., Chamel \& Haensel 2008; Caplan \& Horowitz 2017). Namely, elasticity has imprints in oscillation spectra (e.g., Hansen \& Cioffi 1980; Schumaker \& Thorne 1983; McDermott et al. 1988; Strohmayer et al. 1991; Kozhberov \& Yakovlev 2020) and affects quasi-periodic oscillations, observed after giant flares of soft gamma repeaters (e.g., Gabler et al. 2011, 2012, 2013, 2018;

\footnotetext{
^ E-mail: andr.astro@mail.ioffe.ru
}

Sotani et al. 2018). Elastic stresses can be responsible for asymmetric matter distribution in the crust (so-called mountains; see e.g., Ushomirsky, Cutler \& Bildsten 2000; Haskell, Jones \& Andersson 2006; Horowitz 2010; Johnson-McDaniel \& Owen 2013), which is limited by the material strength of the neutron crust matter (see Horowitz \& Kadau 2009; Chugunov \& Horowitz 2010, 2012; Baiko \& Kozhberov 2017; Baiko \& Chugunov 2018; Kozhberov \& Yakovlev 2020). Having this asymmetry, a rapidly rotating neutron star emits gravitational waves; nowadays gravitational wave observatories put tight upper bounds for amplitude of such waves, constraining thus the asymmetry (e.g., The LIGO Scientific Collaboration et al. 2020). Rapid release of magnetic and elastic energy due to crust failure can be responsible for magnetar bursts Beloborodov \& Levin (2014); Beloborodov \& Li (2016); Li et al. (2016). Crust failure can be also important for pulsar glitches (e.g., Baym \& Pines 1971; Haskell \& Melatos 2015; Akbal \& Alpar 2018; Melatos \& Drummond 2019; Gügercinoğlu \& Alpar 2019; Layek \& Yadav 2020).

The simplest model to study the elastic properties of the neutron star crust and white dwarf core is Coulomb solid model, which considers a solidified system of point charges (atomic nuclei or ions for brevity) on the uniform neutralizing background (e.g., Caplan \& Horowitz 2017). Within this model, the elastic properties of a perfect one-component-bcc lattice at zero temperature were calculated by Fuchs (1936). The finite temperature effects were analyzed by Ogata \& Ichimaru (1990) by Monte Carlo simulations and by Baiko $(2011,2012)$ within phonon formalism, which naturally includes quantum effects. Electron screening was considered 
by Baiko (2012); Kobyakov \& Pethick (2015); Baiko (2015). Elastic properties of multicomponent crystals were studied by Kozhberov (2019).

All these papers provide accurate calculations for monocrystals, which are not isotropic and whose stress-strain tensor depends on more than two independent elastic constants. However, the assumption that the whole neutron star crust (or white dwarf core) is one monocrystal seems to be unrealistic. More likely, it should be polycrystalline (e.g., Kobyakov \& Pethick 2015; Caplan et al. 2018). Authors also suggest an effective shear modulus $\mu_{\text {eff }}$, which should be applicable for macroscopically isotropic polycrystalline matter. In early papers, following Ogata \& Ichimaru (1990), the effective shear modulus was calculated by averaging the shear wave velocity over directions in crystallite. As pointed by Kobyakov \& Pethick (2015), this approach is equal to so-called Voigt average (respective $\mu_{\mathrm{eff}}$ is denoted as $\mu_{\text {eff }}^{\mathrm{V}}$ below), which is based on the assumption that the strain is uniform over all of crystallites; $\mu_{\text {eff }}^{\mathrm{V}}$ known to be an upper limit for $\mu_{\text {eff }}$ (see Blaschke 2017 and section 2.3 for short proof). Kobyakov \& Pethick (2015) suggest to apply the self-consistent theory by Eshelby (1961) and calculate respective shear modulus, which turns to be $\sim 22 \%$ lower than $\mu_{\text {eff }}^{\mathrm{V}}$ for one-component-bcc crystallites.

Unfortunately, as pointed by Landau et al. (1986, section 10, p. $36), \mu_{\text {eff }}$ cannot be calculated accurately, when one has only elastic properties of crystallites, if they are strongly anisotropic (which is the case for one-component Coulomb crystals, e.g., Fuchs 1936; Ogata \& Ichimaru 1990). The explanation is that $\mu_{\mathrm{eff}}$ depends on the correlations in orientations of crystallites. The correlations can depend on the evolution history and, as far as I know, currently they cannot be predicted with high degree of reliability. As a result, one should keep in mind an associated uncertainty in $\mu_{\mathrm{eff}}$, when applying this quantity in astrophysics.

In this letter, I consider general properties of the Coulomb part of the stress-strain tensor $B_{i j k l}$ (roman letters $i, j, k, \ldots$ denote spacial components, see section 2.1 for details) within Coulomb solid model, neglecting ion vibrations (i.e. finite temperature and quantum effects) and assuming the strain to be uniform at the microscopic scale (Voigt appoximation). ${ }^{1}$ I demonstrate the contraction $B_{i j i l}=0$ due to symmetry of the Coulomb interaction (summation over repeated indices is assumed). It is precisely so for the uniform deformation of arbitrary (even multicomponent or/and disordered) The Coulomb solid, which has symmetric stress tensor at undeformed state. Invariance of this convolution with respect to the Voigt average couples the bulk modulus $K$ and $\mu_{\text {eff }}^{\mathrm{V}}$, making only one of them independent. For Coulomb solids, the bulk modulus is determined by the Coulomb (Madelung) energy density of non-deformed state $\epsilon^{\mathrm{C}}$, so that $\mu_{\text {eff }}^{\mathrm{V}}=-(2 / 15) \epsilon^{\mathrm{C}}$ (exactly), which can be also treated as an upper limit for $\mu_{\text {eff }}$. The linear mixing rule combined with ion sphere model leads to the estimate (16), which is applicable for arbitrary composition and structure of the Coulomb solid. For neutron star crusts and white dwarf cores, this estimate can be treated as an upper limit for $\mu_{\text {eff }}$.

\footnotetext{
${ }^{1}$ For astrophysical applications the Coulomb solid model represents only (Coulomb) part of the thermodynamics, which is associated with ions (e.g., Chamel \& Haensel 2008). To obtain the net pressure, stress-strain tensor, etc. one should include contributions associated with degenerate electron gas as well as unbound neutrons in the inner crust of neutron stars. These contributions can be considered as additive and isotropic; they do not affect shear modulus (but dominate for pressure and bulk modulus). Thus, Coulomb contribution can be considered separately.
}

\section{ELASTISITY TENSORS FOR COULOMB CRYSTALS}

\subsection{Elasticity theory at finite pressure}

The elasticity theory describes deformation of solids from some initial (undeformed) state. The deformation can be described by displacement $\boldsymbol{\xi}(\boldsymbol{R})$ of matter elements from position $\boldsymbol{R}$ to $\tilde{\boldsymbol{R}}=\boldsymbol{R}+\boldsymbol{\xi}(\boldsymbol{R})$. For infinitesimal deformations $\xi\left(\boldsymbol{R}^{a}\right)_{i}=u_{i j} R_{j}^{a}$, where $u_{i j}$ is a displacement gradient. Below I consider a uniform deformation, i.e. $u_{i j}$ assumed to be constant over solid (see discussion in section 2.2).

The Coulomb solids have finite pressure in the undeformed state. ${ }^{2}$ It makes the elasticity theory lengthier than the standard textbook version (e.g., Landau et al. 1986) written for a zero pressure, so that several different tensors should be introduced (see below for brief summary and Wallace 1967 for details). ${ }^{3}$ The first one, $S_{i j k l}$ describes the change of the energy $\delta E$ (per unit volume $V$ of undeformed matter), associated with the deformation:

$\delta E=\sigma_{i j} u_{i j}+\frac{1}{2} S_{i j k l} u_{i j} u_{k l}$.

Here $\sigma_{i j}$ is the stress tensor in an undeformed solid, assumed to be isotropic below ( $\sigma=-P \delta_{i j}$, where $P$ is pressure, $\delta_{i j}$ is Kronecker delta). Generally, $S_{i j k l}$ does not have Voigt symmetry (e.g., Wallace 1967).

The tensor $S_{i j k l}$ should be distinguished from the stress-strain tensor $B_{i j k l}=S_{i j k l}-P\left(\delta_{i l} \delta_{j k}-\delta_{i j} \delta_{l k}\right)$, which is used to calculate the change of the stress tensor $\delta \sigma_{i j}$, associated with the deformation

$\delta \sigma_{i j}=\frac{1}{2} B_{i j k l}\left(u_{k l}+u_{l k}\right)$.

Straightforward calculations demonstrate that $B_{i j k l}+B_{i l k j}=S_{i j k l}+$ $S_{i l k j}$. The tensor $B_{i j k l}$ has Voigt symmetry $\left(B_{i j k l}=B_{j i k l}=\right.$ $B_{i j l k}=B_{k l i j}$, see Wallace 1967), and, thus, up to 21 independent elastic parameters.

For isotropic material, the stress-strain tensor $B_{i j k l}^{\mathrm{V}}$ has the same structure as for the elasticity theory at a zero pressure:

$B_{i j k l}^{\mathrm{V}}=K \delta_{i j} \delta_{k l}+\mu_{\mathrm{eff}}^{\mathrm{V}}\left(\delta_{i k} \delta_{j l}+\delta_{i l} \delta_{j k}-\frac{2}{3} \delta_{i j} \delta_{k l}\right)$

giving a common form of the stress-strain relation

$$
\delta \sigma_{i j}=K \delta_{i j} u_{l l}+\mu_{\mathrm{eff}}^{\mathrm{V}}\left(u_{i k}+u_{k i}-\frac{2}{3} \delta_{i k} u_{l l}\right)
$$

2 The pressure is formally negative for Coulomb solids (e.g. Chamel \& Haensel 2008), which does not result in an instability, because the neutralizing background is considered as incompressible. For astrophysical applications, the stability is associated with positive pressure of electron gas (and unbound neutrons in the inner crust of neutron stars).

3 Marcus, Ma \& Qiu (2002) suggest that the theory of elasticity at finite pressure can be simplified and unified by the use of the Gibbs free energy (see, however, the comment by Steinle-Neumann \& Cohen 2004 and reply by Marcus \& Qiu 2004 for discussion of crucial details). In particular, the approach by Marcus et al. (2002) allows to deal only with the Voigt symmetric tensors. However, at least for some first-principle calculations (e.g. Baiko 2011, 2012; Kozhberov 2019 and this work) the approach by Wallace (1967) seems to be more useful because it shortens derivations. 


\subsection{Symmetry of the elasticity tensor for Coulomb crystals}

To derive $S_{i j k l}$ tensor, I calculate a change of energy associated with deformation. The energy of the Coulomb solid can be written as

$$
\begin{aligned}
E & =\sum_{a} \sum_{b>a} \frac{Z^{a} Z^{b} e^{2}}{\left|\boldsymbol{R}^{a}-\boldsymbol{R}^{b}\right|}-\sum_{a} \int \frac{Z^{a} e^{2} n_{\mathrm{e}}}{\left|\boldsymbol{R}^{a}-\boldsymbol{r}\right|} \mathrm{d}^{3} \boldsymbol{r} \\
& +\frac{1}{2} \iint \frac{e^{2} n_{\mathrm{e}}^{2}}{\left|\boldsymbol{r}-\boldsymbol{r}^{\prime}\right|} \mathrm{d}^{3} \boldsymbol{r} \mathrm{d}^{3} \boldsymbol{r}^{\prime} .
\end{aligned}
$$

Here upper indices $a$ and $b$ enumerate ions, $Z^{a} e$ and $\boldsymbol{R}^{a}$ are the position and the charge of ion $a$ respectively ( $e$ is an absolute value of electron charge). The electron number density is uniform; $n_{\mathrm{e}}(\boldsymbol{r})=$ $\sum_{a} Z^{a} / V$ due to quasineutrality condition ( $V$ is volume). In equation (5) terms are ion-ion, ion-electron, and electron-electron interaction energies respectively. The ion positions after the deformation are

$\tilde{R}_{i}^{a}=R_{i}^{a}+u_{i j} R_{j}^{a}$.

The same deformation is applied for electrons. As a result, their number density becomes $\tilde{n}_{e}=n_{e} / J$, where $J$ is Jacobian of $\boldsymbol{r} \rightarrow \tilde{\boldsymbol{r}}$ transformation (the quasineutrality condition obviously holds true after deformation). The energy in the deformed state is

$$
\begin{aligned}
\tilde{E} & =\sum_{a} \sum_{b>a} \frac{Z^{a} Z^{b} e^{2}}{\left|\tilde{\boldsymbol{R}}^{a}-\tilde{\boldsymbol{R}}^{b}\right|}-\sum_{a} \int \frac{Z_{i} e^{2} \tilde{n}_{\mathrm{e}}}{\left|\tilde{\boldsymbol{R}}^{a}-\tilde{\boldsymbol{r}}\right|} \mathrm{d}^{3} \tilde{\boldsymbol{r}} \\
& +\frac{1}{2} \iint \frac{e^{2} \tilde{n}_{\mathrm{e}}^{2}}{\left|\tilde{\boldsymbol{r}}-\tilde{\boldsymbol{r}}^{\prime}\right|} \mathrm{d}^{3} \tilde{\boldsymbol{r}} \mathrm{d}^{3} \tilde{\boldsymbol{r}}^{\prime} .
\end{aligned}
$$

Formal change of variables in all integrals from $\tilde{\boldsymbol{r}}$ to $\boldsymbol{r}$ leads to appearance of a Jakobian factor $J$, which finally disappears, because $J \tilde{n}_{e}=n_{e}$. Thus, the change of the energy associated with the deformation can be written as

$$
\begin{aligned}
\delta E & =\tilde{E}-E=\sum_{a} \sum_{b>a} Z^{a} Z^{b} e^{2}\left(\frac{1}{|\tilde{\Delta}|}-\frac{1}{|\Delta|}\right) \\
& -\sum_{a} \int Z_{i} e^{2} n_{\mathrm{e}}\left(\frac{1}{|\tilde{\Delta}|}-\frac{1}{|\Delta|}\right) \mathrm{d}^{3} \boldsymbol{r} \\
& +\frac{1}{2} \iint e^{2} n_{\mathrm{e}}^{2}\left(\frac{1}{|\tilde{\Delta}|}-\frac{1}{|\Delta|}\right) \mathrm{d}^{3} \boldsymbol{r} \mathrm{d}^{3} \boldsymbol{r}^{\prime} .
\end{aligned}
$$

Here and below in the first line $\Delta=\boldsymbol{R}^{a}-\boldsymbol{R}^{b}$, in the second line $\boldsymbol{\Delta}=\boldsymbol{R}^{a}-\boldsymbol{r}$, and, in the third line $\boldsymbol{\Delta}=\boldsymbol{r}-\boldsymbol{r}^{\prime}$. Similar notations are applied for quantities in the deformed state, e.g., $\tilde{\boldsymbol{\Delta}}=\tilde{\boldsymbol{R}}^{a}-\tilde{\boldsymbol{R}}^{b}$ for the first line.

The Taylor expansion over $\boldsymbol{\xi}$ for terms in parenthesis in the equation (8) gives:

$$
\frac{1}{|\tilde{\Delta}|}-\frac{1}{|\Delta|} \approx \frac{\Delta_{i} \Delta_{j}}{\Delta^{3}} u_{i j}+\frac{3 \Delta_{i} \Delta_{k}-\Delta^{2} \delta_{i k}}{\Delta^{5}} \Delta_{j} \Delta_{l} u_{i j} u_{k l}
$$

Comparison with the equation (1) gives:

$$
\begin{aligned}
\sigma_{i j} & =\sum_{a} \sum_{b>a} Z^{a} Z^{b} e^{2} \frac{\Delta_{i} \Delta_{j}}{\Delta^{3}}-\sum_{a} \int Z_{i} e^{2} n_{\mathrm{e}} \frac{\Delta_{i} \Delta_{j}}{\Delta^{3}} \mathrm{~d}^{3} \boldsymbol{r} \\
& +\frac{1}{2} \iint e^{2} n_{\mathrm{e}}^{2} \frac{\Delta_{i} \Delta_{j}}{\Delta^{3}} \mathrm{~d}^{3} \boldsymbol{r} \mathrm{d}^{3} \boldsymbol{r}^{\prime} \\
S_{i j k l} & =\sum_{a} \sum_{b>a} Z^{a} Z^{b} e^{2} \frac{3 \Delta_{i} \Delta_{k}-\Delta^{2} \delta_{i k}}{\Delta^{5}} \Delta_{j} \Delta_{l} \\
& -\sum_{a} \int Z_{i} e^{2} n_{\mathrm{e}} \frac{3 \Delta_{i} \Delta_{k}-\Delta^{2} \delta_{i k}}{\Delta^{5}} \Delta_{j} \Delta_{l} \mathrm{~d}^{3} \boldsymbol{r} \\
& +\frac{1}{2} \iint e^{2} n_{\mathrm{e}}^{2} \frac{3 \Delta_{i} \Delta_{k}-\Delta^{2} \delta_{i k}}{\Delta^{5}} \Delta_{j} \Delta_{l} \mathrm{~d}^{3} \boldsymbol{r} \mathrm{d}^{3} \boldsymbol{r}^{\prime} .
\end{aligned}
$$

To calculate $S_{i j i l}=S_{i j k l} \delta_{i k}$, I perform the contraction before the summation or/and integration: each term contains $\left(3 \Delta_{i} \Delta_{k}-\right.$ $\left.\Delta^{2} \delta_{i k}\right) \delta_{i k} \equiv 0$, thus $S_{i j i l}=0 .{ }^{4}$ In particular, $S_{i j i j}=0$ (for cubic symmetry this form is equivalent to the previous). It is easy to show that $B_{i j i l}=0$ (in particular, $\left.B_{i j i j}=0\right)$.

In this section I do not appeal to any assumption on a structure or a composition, thus the results can be applied for multicomponent Coulomb solids with arbitrary structure (crystalline or disordered).

It is worth to warn the reader that in this section I assume that the deformation is uniform at the microphysical level, i.e. displacement of all ions is given by equation (6). This assumption seems natural, especially when considering deformation of crystals (e.g., Baiko 2015). However, generally it can be violated - for given macroscopic deformation (e.g., applied at the boundary) the deformation field within the solid can be non-uniform, if it is energeticaly favorable (i.e. if it leads to a change of the energy lower than given by the equation 7). In this case, the actual $S_{i j k l}$ tensor corresponds to lower energy and relations $S_{i j i l}=0$ and $B_{i j i l}=0$ can be violated.

\subsection{Shear modulus for isotropic material: the Voigt average}

Here I apply the well known Voigt average approach (e.g. Blaschke 2017) to estimate the elastic properties of the polycrystalline matter. The approach is based on the assumption that the strains are equal for all crystallites, leading to the second-order change in energy in the form

$\delta^{(2)} E=\sum_{c} \frac{V_{c}}{2 V} S_{i j k l}^{c} u_{i j} u_{k l}=S_{i j k l}^{\mathrm{V}} u_{i j} u_{k l}$,

where summation is performed over crystallites, $V_{c}$ and $S_{i j k l}^{c}=$ $S_{\text {mnop }} R_{i m}^{c} R_{j n}^{c} R_{k o}^{c} R_{l p}^{c}$ are volume and elastic tensors for the crystallite $c$. Here rotation matrix $R_{i m}^{c}$ is applied to transform the original frame to the crystal frame. $S_{i j k l}^{\mathrm{V}}$ corresponds to the Voigt averaged elastic tensor. The Voigt average gives an upper limit for $\mu_{\text {eff }}$ due to the same reasons as discussed at the end of the previous section: it assumes the uniform deformation for all crystallites, but non-uniform deformation, in principle, can decrease the energy and, subsequently, lead to a lower actual value of $\mu_{\text {eff }}$. Two contractions are invariant with respect to Voigt average: $S_{i i j j}^{\mathrm{V}}=S_{i i j j}$ and $S_{i j i j}^{\mathrm{V}}=S_{i j i j}$ (e.g., Blaschke 2017; the invariance follows from $R_{i k} R_{i l}=\delta_{k l}$, see e.g., Varshalovich, Moskalev \& Khersonskii 1988). As far as the second one vanishes for Coulomb solids, it should also vanish for the Voigtaveraged stress-strain tensor: $S_{i j i j}^{\mathrm{V}}=0$.

It is worth to point, that $S_{i j i j}^{\mathrm{V}}=0$ can be derived directly within the approach of the section 2.2 considering deformation of polycrystalline matter as a whole.

Contraction $S_{i j i j}^{\mathrm{V}}=0$ implies $B_{i j i j}^{\mathrm{V}}=0$. Combined with general form of $B_{i j k l}$ for isotropic material (equation 3 ) it imposes condition

$\mu_{\mathrm{eff}}^{\mathrm{V}}=-\frac{3}{10} K=-\frac{2}{15} E^{\mathrm{M}}$,

which should hold true exactly. In the last equality I use $K=4 \epsilon^{\mathrm{M}} / 9$, which follows from scaling of the Madelung energy density $\epsilon^{\mathrm{M}} \propto$ $n_{\mathrm{e}}^{4 / 3}$. As far as $\epsilon^{\mathrm{M}}$ is negative (see footnote 2), $\mu_{\mathrm{eff}}^{\mathrm{V}}$ is positive.

Let me check the equation (13) by previous calculations. To begin

4 This result can not be straightforwardly generalized for general pow-law
potential $\propto 1 / r^{n}$, because for this potential the respective term becomes
$\left[n(n+2) \Delta_{i} \Delta_{k}-n \Delta^{2} \delta_{i k}\right] \delta_{i k}=n(n-1) \Delta^{2} \neq 0$ for $n \neq 1$. 
with, the Madelung energy for one-component-bcc crystal was calculated by Baiko et al. (2001): $\epsilon^{\mathrm{M}}=-0.895929255682 Z^{5 / 3} e^{2} n_{\mathrm{Z}} / a_{\mathrm{e}}$, where $a_{\mathrm{e}}=\left[3 /\left(4 \pi n_{\mathrm{e}}\right)\right]^{1 / 3}$ is electron sphere radius and $n_{\mathrm{Z}}$ is number density of ions with charge $\mathrm{Ze}$. According to equation (13):

$\mu_{\text {eff, bcc }}^{\mathrm{V}}=0.119457234091 \frac{Z^{5 / 3} e^{2}}{a_{\mathrm{e}}} n_{Z}$.

It perfectly agrees with the result by Baiko (2011), where the coefficient 0.1194572 was given. For two-component ordered crystals, tensor $S_{i j k l}$ was calculated by Kozhberov (2019) and $S_{i j i j}=0$ (within this calculation's accuracy). ${ }^{5}$

Similarly, the equation (13) can be straightforwardly applied to calculate $\mu_{\mathrm{eff}}^{\mathrm{V}}$ for multicomponent lattice structures, whose energies were calculated by Chamel \& Fantina (2016); Kozhberov (2018), but $\mu_{\text {eff }}^{\mathrm{V}}$ was not studied previously.

Even more important is the fact that the equation (13) allows me to write down an universal estimate for $\mu_{\text {eff }}^{\mathrm{V}}$ using the ion-sphere model by Salpeter (1954) combined with linear mixing rule (e.g., Hansen \& Vieillefosse 1976; Ogata et al. 1993). This combination describes Madelung energy of crystals very accurately (Ogata et al. 1993; Kozhberov 2019) and leads to

$\epsilon^{\mathrm{M}} \approx-\frac{9}{10} \sum_{Z} \frac{Z^{5 / 3} e^{2}}{a_{\mathrm{e}}} n_{Z}$.

According to the equation (13):

$\mu_{\mathrm{eff}}^{\mathrm{V}} \approx-\frac{3}{25} \sum_{Z} \frac{Z^{5 / 3} e^{2}}{a_{\mathrm{e}}} n_{Z}=0.12 \sum_{Z} \frac{Z^{5 / 3} e^{2}}{a_{\mathrm{e}}} n_{Z}$.

For one-component-bcc it deviates from the accurate calculations by (14) for mere $0.05 \%$.

The results of this section (equations 13 and 16) are applicable for amorphous Coulomb solids, which are generally isotropic and do not require Voigt average.

\section{SUMMARY AND DISCUSSION}

I consider elastic properties of neutron star crust matter within the Coulomb solid model (solidified system of point charges (ions) on the uniform unpolarizable neutralizing background). The results are also applicable for white dwarf cores and other systems, which can be described by Coulomb solid model (e.g., dusty plasma).

Neglecting thermal and zero-point vibrations of ions and assuming uniform strain, I derive universal exact relation for the Coulomb part of the stress-stain tensor $B_{i j i l}=0$ (also for tensor in equation 1: $S_{i j i l}=0$ ). This result does not depend on the structure and composition of the solid.

I apply this result to consider elastic properties of macroscopically isotropic polycrystalline matter using Voigt average and demonstrate that the effective shear modulus is determined by the Madelung energy density $\epsilon^{\mathrm{M}}: \mu_{\text {eff }}^{\mathrm{V}}=-(2 / 15) \epsilon^{\mathrm{M}}$ (again, exactly and for arbitrary structure and composition). I check this relation by comparison with previously calculated values for one- and two-component crystals. Using the ion sphere model by Salpeter (1954) and linear mixing rule to estimate Madelung energy, I suggest a simple estimate for $\mu_{\mathrm{eff}}^{\mathrm{V}}$ (equation 16). The results are also directly applicable for isotropic amorphous solids.

5 In fact, some miraculous coincidences in the numerical results by Kozhberov (2019) were the starting point for this study.
The Voigt average is equal to averaging of the dispersion relations for long-wavelength transversal modes (approach suggested by Ogata \& Ichimaru 1990) and thus $\mu_{\mathrm{eff}}^{\mathrm{V}}$ can be applied to calculate root-mean-square velocity of these modes in monocrystals. For amorphous solids, which are isotropic, equation (16) allows to consider long-wavelength transversal modes and thus discuss their low-temperature thermodynamics.

Universal estimate (16) suggests that the elastic properties should vary rather smoothly within neutron star crust, at least if there are no rapid changes of composition. This result is important for torsional oscillations of neutron star crust (e.g. Kozhberov \& Yakovlev 2020). Equation (16) also supports all previous neutron star models based on the effective shear modulus of one-component-bcc crystal: their numerical results are valid for arbitrary microscopic structure of the crust, because $\mu_{\text {eff }}^{\mathrm{V}}$ depends on it weakly.

It is worth to warn the reader, that all results in this letter were obtained assuming uniform deformation at the microphysical level (i.e., displacement of all ions is given by equation 6). As discussed at the end of section 2.2, this assumptions can be violated, if it allows the lower energy at the same (macroscopic) strain. In this case $S_{i j i l}$ can be non-zero. In particular, for polycrystalline and amorphous matter the estimate (16) should be considered as an upper limit for the effective shear modulus (the Voigt average known to give an upper limit for the effective shear modulus). However, as follows from Kozhberov (2019), multicomponent Coulomb crystals tend to be less anisotropic than one-component crystals and different estimates of $\mu_{\text {eff }}$ become closer to $\mu_{\text {eff }}^{V}$. The ion vibrations and electron screening are neglected in this work. They decrease the shear modulus (e.g., Baiko 2012), thus equation (16) gives an upper bound for $\mu_{\text {eff }}$ even if these effects are included.

The approach of the section 2.2 can be easily generalized for the non-spherical nuclei (so-called pasta phases) in the bottom of the inner crust of neutron star by introducing proton charge density with respective integration instead of summation over point-like ions. I plan to study the applicability of such model for pasta phases in subsequent publication.

Similarly, the result of the section 2.2 can be applied in electrostatics: the variation of electrostatic energy of a body with a given charge distribution under uniform deformation can be described by an analogue of the equation (1), where contraction $S_{i j i l}=0$.

\section{ACKNOWLEDGEMENTS}

I'm grateful to A.A. Kozhberov, who provided me with an unpublished (at that time) version of Kozhberov (2019). I also thank anonymous referee for the fast and constructive report. This research was partially supported by The Ministry of Science and Higher Education of the Russian Federation (Agreement with Joint Institute for High Temperatures RAS No 075-15-2020-785).

\section{DATA AVAILABILITY}

The data underlying this letter are available in the letter.

\section{REFERENCES}

Akbal O., Alpar M. A., 2018, MNRAS, 473, 621

Baiko D. A., 2002, Phys. Rev. E, 66, 056405

Baiko D. A., 2011, MNRAS, 416, 22

Baiko D. A., 2012, Contributions to Plasma Physics, 52, 157 
Baiko D. A., 2015, MNRAS, 451, 3055

Baiko D. A., Chugunov A. I., 2018, MNRAS, 480, 5511

Baiko D. A., Kozhberov A. A., 2017, MNRAS, 470, 517

Baiko D. A., Potekhin A. Y., Yakovlev D. G., 2001, Phys. Rev. E, 64, 057402

Baym G., Pines D., 1971, Annals of Physics, 66, 816

Beloborodov A. M., Levin Y., 2014, ApJ, 794, L24

Beloborodov A. M., Li X., 2016, ApJ, 833, 261

Blaschke D. N., 2017, Journal of Applied Physics, 122, 145110

Brush S. G., Sahlin H. L., Teller E., 1966, J. Chem. Phys., 45, 2102

Caplan M. E., Horowitz C. J., 2017, Rev. Mod. Phys., 89, 041002

Caplan M. E., Cumming A., Berry D. K., Horowitz C. J., Mckinven R., 2018, ApJ, 860, 148

Carreau T., Gulminelli F., Chamel N., Fantina A. F., Pearson J. M., 2020a, A\&A, 635, A84

Carreau T., Fantina A. F., Gulminelli F., 2020b, A\&A, 640, A77

Chamel N., Fantina A. F., 2016, Phys. Rev. C, 94, 065802

Chamel N., Haensel P., 2008, Liv. Rev. Relativ., 11, 10

Chugunov A. I., Horowitz C. J., 2010, MNRAS, 407, L54

Chugunov A. I., Horowitz C. J., 2012, Contributions to Plasma Physics, 52,122

Eshelby J. D., 1961, Prog. Solid Mech., 2, 87

Fantina A. F., De Ridder S., Chamel N., Gulminelli F., 2020, A\&A, 633, A149

Fuchs K., 1936, Proc. of the Royal Society of London A: Mathematical, Physical and Engineering Sciences, 153, 622

Gabler M., Cerdá Durán P., Font J. A., Müller E., Stergioulas N., 2011, MNRAS, 410, L37

Gabler M., Cerdá-Durán P., Stergioulas N., Font J. A., Müller E., 2012, MNRAS, 421, 2054

Gabler M., Cerdá-Durán P., Font J. A., Müller E., Stergioulas N., 2013, MNRAS, 430, 1811

Gabler M., Cerdá-Durán P., Stergioulas N., Font J. A., Müller E., 2018, MNRAS, 476, 4199

Gügercinoğlu E., Alpar M. A., 2019, MNRAS, 488, 2275

Haensel P., Potekhin A., Yakovlev D., 2006, Neutron Stars 1: Equation of State and Structure. Astrophysics and Space Science Library, Springer-Verlag, Berlin

Hamaguchi S., Farouki R. T., Dubin D. H. E., 1997, Phys. Rev. E, 56, 4671

Hansen C. J., Cioffi D. F., 1980, ApJ, 238, 740

Hansen J. P., Vieillefosse P., 1976, Phys. Rev. Lett., 37, 391

Haskell B., Melatos A., 2015, International Journal of Modern Physics D, 24,1530008

Haskell B., Jones D. I., Andersson N., 2006, MNRAS, 373, 1423

Horowitz C. J., 2010, Phys. Rev. D, 81, 103001

Horowitz C. J., Kadau K., 2009, Physical Review Letters, 102, 191102

Johnson-McDaniel N. K., Owen B. J., 2013, Phys. Rev. D, 88, 044004

Jones P. B., 1999, Phys. Rev. Lett., 83, 3589

Jones M. D., Ceperley D. M., 1996, Phys. Rev. Lett., 76, 4572

Kobyakov D., Pethick C. J., 2015, MNRAS, 449, L110

Kozhberov A., 2018, Phys. Rev. E, 98, 063205

Kozhberov A. A., 2019, MNRAS, 486, 4473

Kozhberov A. A., Yakovlev D. G., 2020, MNRAS,

Landau L., Lifshitz E., Kosevich A., Pitaevskiĭ L., 1986, Theory of Elasticity. Course of theoretical physics, Butterworth-Heinemann

Layek B., Yadav P., 2020, arXiv e-prints, p. arXiv:2009.08085

Li X., Levin Y., Beloborodov A. M., 2016, ApJ, 833, 189

Marcus P. M., Qiu S. L., 2004, Journal of Physics: Condensed Matter, 16, 8787

Marcus P. M., Ma H., Qiu S. L., 2002, Journal of Physics: Condensed Matter, 14, L525

McDermott P. N., van Horn H. M., Hansen C. J., 1988, ApJ, 325, 725

Medin Z., Cumming A., 2010, Phys. Rev. E, 81, 036107

Melatos A., Drummond L. V., 2019, ApJ, 885, 37

Ogata S., Ichimaru S., 1990, Phys. Rev. A, 42, 4867

Ogata S., Ichimaru S., van Horn H. M., 1993, ApJ, 417, 265

Potekhin A. Y., Chabrier G., 2000, Phys. Rev. E, 62, 8554

Rezzolla L., Pizzochero P., Jones D. I., Rea N., Vidaña I., eds, 2018, The Physics and Astrophysics of Neutron Stars. Springer, Cham, Switzerland Salpeter E. E., 1954, Australian Journal of Physics, 7, 373
Sauls J. A., Chamel N., Alpar M. A., 2020, arXiv e-prints, p. arXiv:2001.09959

Schumaker B. L., Thorne K. S., 1983, MNRAS, 203, 457

Sotani H., Iida K., Oyamatsu K., 2018, MNRAS, 479, 4735

$\begin{array}{llll}\text { Steinle-Neumann } \quad \text { G., Cohen R. } & \text { E., }\end{array}$ Journal of Physics: Condensed Matter, 16, 8783

Strohmayer T., Ogata S., Iyetomi H., Ichimaru S., van Horn H. M., 1991, ApJ, 375,679

The LIGO Scientific Collaboration et al., 2020, arXiv e-prints, p. arXiv:2007.14251

Ushomirsky G., Cutler C., Bildsten L., 2000, MNRAS, 319, 902

Varshalovich D., Moskalev A., Khersonskii V., 1988, Quantum Theory of Angular Momentum. World Scientific Pub., Singapore, https://books.google.ru/books?id=nXcGCwAAQBAJ

Wallace D. C., 1967, Physical Review, 162, 776

This paper has been typeset from a $\mathrm{T}_{\mathrm{E}} \mathrm{X} / \mathrm{L} \mathrm{T} \mathrm{E} \mathrm{X}$ file prepared by the author. 